Yu Yang • Hes Ondrej · Lanjing Zhang • Jian Lu •

Min Lu · Hua Wang · Jie Zheng • Michal Michal

\title{
Mixed epithelial and stromal tumor of the kidney with cervical and intestinal differentiation
}

Received: 20 February 2005 / Accepted: 3 April 2005 / Published online: 16 July 2005

(C) Springer-Verlag 2005

Dear Editors:

Mixed epithelial and stromal tumor of the kidney (MESTK) has only recently been recognized as a rare benign tumor of the kidney which has distinct clinical and morphological characteristics. The name MESTK was first used by Michal and Syrucek in 1998 [1], and was newly recognized in the recent WHO classification of the urinary system as a distinct entity [2].

We report a unique case of MESTK which showed cervical and intestinal epithelial differentiation. To the best of our knowledge, no such differentiation within MESTK has ever been described in the literature [3, 4]. A 50-yearold woman presented with hematuria associated with pain in the left flank. The patient had a 20-year history of taking oral contraceptives. Computed tomography revealed a

Y. Yang $\cdot$ M. Lu $\cdot$ H. Wang $\cdot$ J. Zheng $(\bowtie)$

Department of Pathology,

Health Science Center,

Peking University,

38 Xue-yuan Road,

100083 Beijing, China

e-mail: zhengjie@bjmu.edu.cn

Tel.: +86-010-82801731

Fax: $+86-010-82801731$

H. Ondrej · M. Michal

Sikl's Department of Pathology,

Charles University Hospital,

Pilsen, Czech Republic

\section{Zhang}

Department of Internal Medicine,

University of Michigan

Medical School,

Ann Arbor, MI, USA

J. Lu

Department of Urology,

3rd Hospital,

Peking University,

Beijing, China
$7 \mathrm{~cm}$ solid and cystic mass at the upper poles of the left kidney. On thorough clinical check-up, no other tumor was found in the patient. Under the diagnosis of renal tumor, the patient underwent total nephrectomy and adrenalectomy. After surgery, no additional treatment was given. Seven months after excision of the tumor, the patient is without signs of recurrence and metastasis. Macroscopically, the tumor was localized in the upper part of the kidney. The tumor was $7 \mathrm{~cm}$ in diameter, well-circumscribed, and composed of irregular mixtures of solid and cystic areas. The solid areas of the tumor were firm and gray-white on gross section. The cysts varied in size ranging from 0.5 to $2 \mathrm{~cm}$ in diameter. There were no signs of infiltrative growth into the surrounding renal parenchyma and the adjacent calyceal system.

Histologically, the tumor was composed of a mixture of epithelial elements that formed glands and cysts and stromal component formed mainly by spindle cells. The epithelial component had a characteristic appearance both

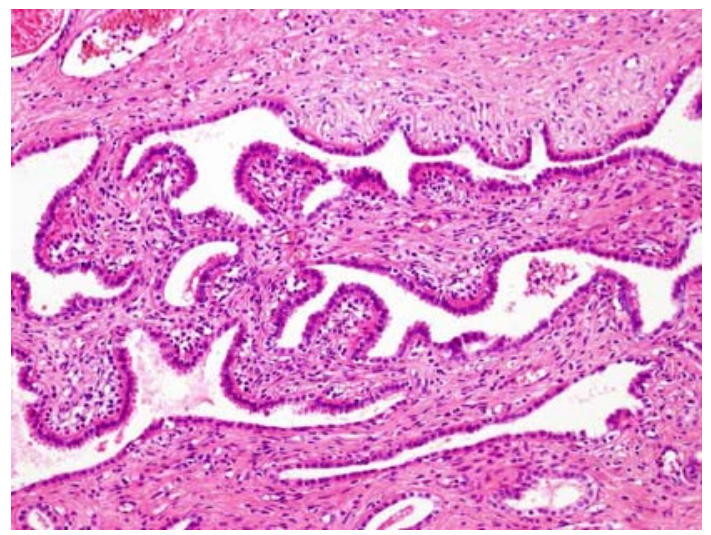

Fig. 1 Glandular epithelium formed serous tubules and multilocular cysts lined by cells with ciliated brush border and peg cells typical of Fallopian tubes. The stroma has bland looking spindle cell appearance, typical of MESTKs 


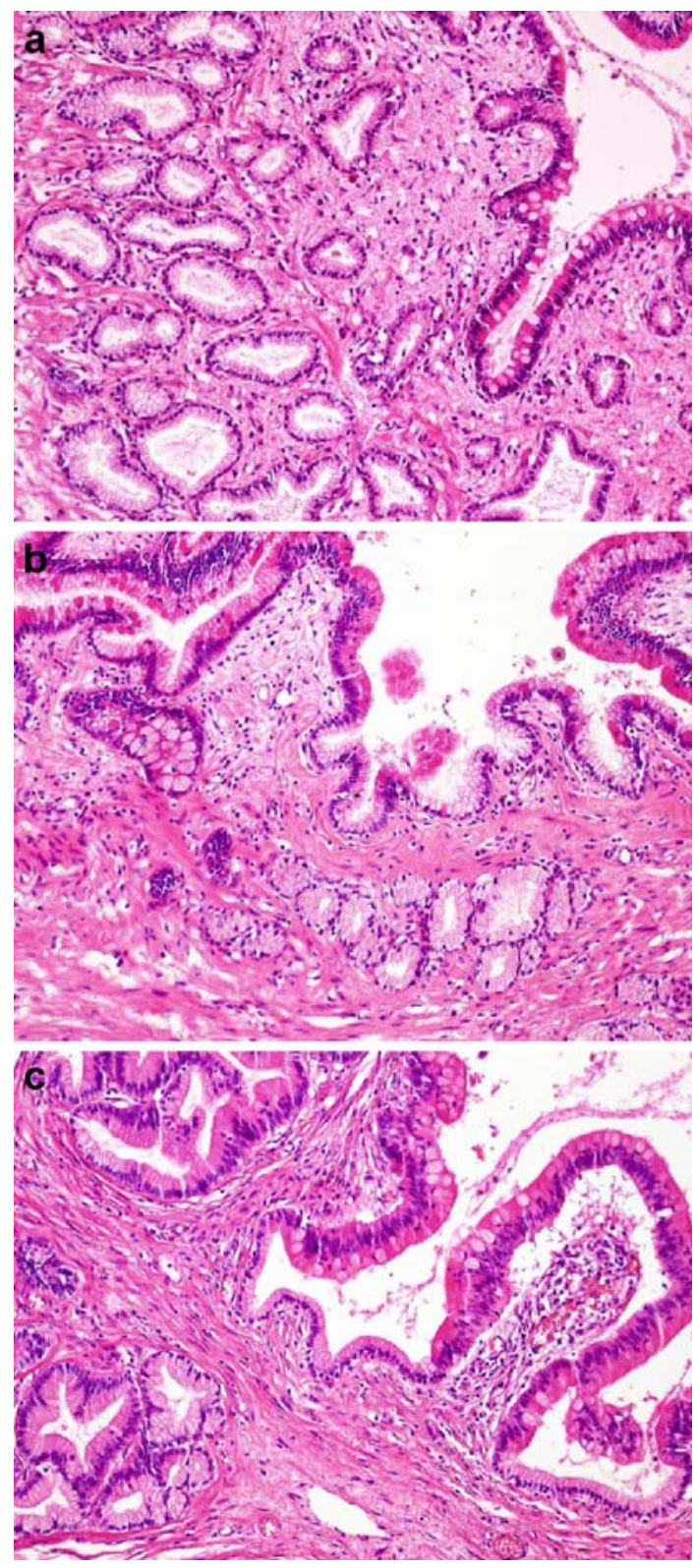

Fig. 2 In some places, the adenomatous structures had the appearance of cervical glands (left side) and intestinal (right side) (a). Focally Paneth cell differentiation was seen in the epithelium of the MESTK (b). The border between the intestinal and pyloric epithelium was often sharp (c)

in architecture and cell type. In some places, glandular epithelium formed serous tubules and multilocular cysts lined by ciliated brush border and peg cells typical of Fallopian tubes (Fig. 1). In other places, the adenomatous structures had the appearance of cervical glands (Fig. 2a) and still other areas revealed typical intestinal epithelium (Fig. 2a) containing Paneth (Fig. 2b) and goblet cells. The border between the intestinal and cervical epithelium was often sharp (Fig. 2c). No cytological atypia or mitotic fig-

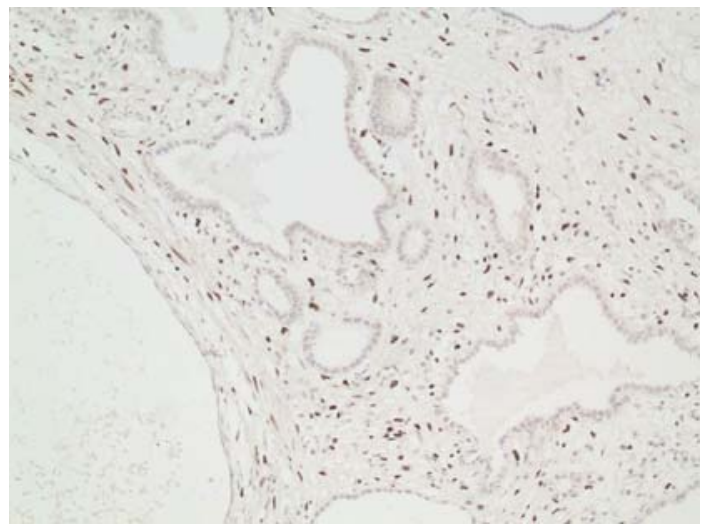

Fig. 3 The stromal elements were immunoreactive to ER

ures were observed in the epithelial component. The excised adrenal gland was normal without signs of tumor. Immunohistochemically, the epithelial elements expressed cytokeratin (dilution 1:50; Dako, Denmark) and were immunonegative for ER (dilution 1:50; Dako), PR (dilution 1:50; Dako). The stromal elements were focally ER (Fig. 3), PR and smooth muscle actin (SMA) (dilution 1: 50; Dako) positive. Both the epithelial and stromal elements failed to react with S-100 (dilution 1:50; Dako, Denmark), CD34 (dilution 1:50; Dako), HMB-45 (dilution 1:50; Dako) antibodies. Considering the clinicopathological features, a diagnosis of MESTK was easily established in our case $[3,4]$.

While our case demonstrated morphological features typical for MESTKs, it also showed unique glandular structures represented by cervical and intestinal mucinous epithelium containing goblet and Paneth cells, which is a hitherto undescribed differentiation in MESTK. The presence of cervical and intestinal mucinous epithelia in MESTK is a hitherto undescribed feature of MESTK. The cervical and intestinal mucinous cystic tumors belong to the most common types of epithelial neoplasms occurring in the ovary. In addition, transition between cervical and intestinal epithelium containing goblet cells within single glandular structures is a well known phenomenon described in ovarian tumors [5]. Since MESKT is a tumor displaying a wide range of epithelia and the stroma are often indistinguishable from that of the ovary, it is not surprising that the same transitions between cervical and intestinal epithelium containing goblet cells within single glandular structures were observed in our case. The description of intestinal epithelium in MSTK is important, because it must be differentiated from metastatic mucinous well-differentiated cystadenocarcinomas originating in intestinal tract, gallbladder and pancreas to kidney. This differentiation is easy, because metastatic adenocarcinomas with intestinal differentiation do not reveal other types of epithelium as cervical or that of Fallopian tubes and spindle cell stroma as seen in our case. 


\section{References}

1. Michal M, Syrucek M (1998) Benign mixed epithelial and stromal tumour of the kidney. Pathol Res Pract 194:445-448

2. Eble JN, Sauter G, Epstein JI, Sesterhenn IA (2004) World health organization classification of tumors. Pathology and genetics of tumors of the urinary system and male genital organs. IARC Press, Lyon

3. Michal M, Hes O, Bisceglia M, Simpson RHW, Spagnolo DV, Parma A, Boudova L, Hora M, Zachoval R, Suster S (2004) Mixed epithelial and stromal tumors of the kidney. A report of 22 cases. Virchows Arch 445:359-367
4. Adsay NV, Eble JN, Srigley JR, Jones EC, Grignon DJ (2000) Mixed epithelial and stromal tumor of the kidney. Am J Surg Pathol 24:958-970

5. Scully RE, Young RH, Clement PB (1996) Atlas of tumor pathology. Tumors of the ovary, maldeveloped gonads, fallopian tube and broad ligament. Armed Forces Institute of Pathology, Washington, D.C. 